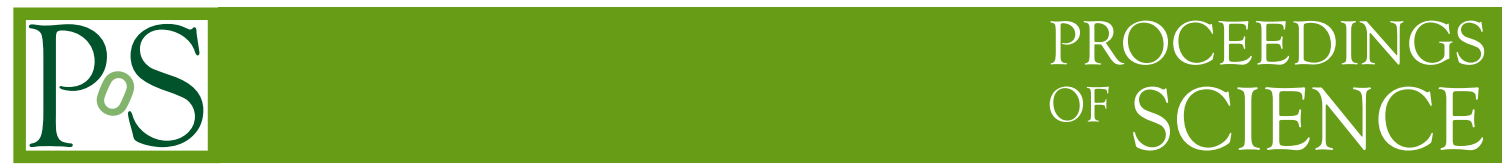

\title{
Performance of the ATLAS trigger and DAQ system
}

\author{
Ellie Dobson*† \\ CERN \\ E-mail: eleanor.dobsonecern.ch
}

The ATLAS Trigger and Data Acquisition (TDAQ) system is an essential part of the ATLAS experiment at the LHC. It is responsible for reducing the event rate from the design bunch-crossing rate of $40 \mathrm{MHz}$ to an output recording rate of $200-400 \mathrm{~Hz}$. We will discuss the achievements of the ATLAS TDAQ system during the 2010 and 2011 data taking periods, with an overview of the challenges and plans for adapting to the upcoming upgrade of the LHC.

The 2011 Europhysics Conference on High Energy Physics-HEP 2011,

July 21-27, 2011

Grenoble, Rhône-Alpes France

\footnotetext{
* Speaker.

$\dagger$ on behalf of the ATLAS Collaboration
} 


\section{Overview of the system}

The ATLAS trigger is designed to select signal-like events from a large background in three levels: a first-level (L1) implemented in custom-built electronics, as well as the two levels of the high level trigger (HLT) software triggers executed on large computing farms.

The first-level trigger is comprised of calorimeter, muon and forward triggers to identify event features such as missing transverse energy, as well as candidate electrons, photons, jets and muons. Input signals from these objects are processed by the L1 Central Trigger to form a L1 Accept (L1A) decision. L1A and timing information are sent to all sub-detectors, which push their data to DAQ buffers. The first part of the HLT system (called Level 2) pulls the data from the buffers on demand, while the second part (called Event Filter) works with the whole event at hand.

All the software required to configure and control the ATLAS data taking is the responsibility of the control and configuration system. This system features a great scalability against the always increasing number of software processes in the TDAQ system (corresponding to the growth of the HLT farm). Several critical actions are performed without the intervention of the operator, something that greatly enhances the experiment's data taking reliability and efficiency.

\section{Data taking efficiency}

Since the end of 2010, the LHC has provided ${ }^{1}$ an increase in instantaneous luminosity from $10^{32}$ to $10^{33} \mathrm{~cm}^{-2} \mathrm{~s}^{-1}$. The average number of interactions per bunch crossing has increased from 2 to 7 , and the bunch spacing from 75 to $50 \mathrm{~ns}$. Despite these increasingly demanding conditions, the data taking effiency has remained stable at $94.5 \%$.

The L1 trigger may veto events (after a trigger has fired, or when a sub-detector readout is busy for example) to protect the front-end readout buffers from overflowing. L1 dead time is a measure of how efficient the data-taking has been. Figure 2 shows the rate of dead time before and after the calorimeter noise thresholds were raised. It shows that the raising of the noise threshold reduced the dead time, by decreasing the number of cells entering the processing and thus the processing time .

To maximise data taking efficiency, trigger rates may also be dynamically controlled during a particular run by adjusting prescales - the percentage of times a given trigger is vetoed - without need for stop and restart, which also reduces the amount of dead time. In addition the trigger rate may be further controlled as luminosity increases by adjusting the tightness of the trigger selection.

\section{Preparation for evolving $\mathrm{LHC}$ conditions}

In normal running, some data are recorded in a way such that it is not trigger-biased. The trigger may then be re-run offline on these data to predict system evolution as pileup and luminosity increase. Figure 3 shows the rates that were predicted, compared to those which were actually recorded at a higher luminosity. It demonstrates the ability to predict future rates in this manner.

\footnotetext{
${ }^{1}$ numbers quoted are those at the time of the conference talk
} 

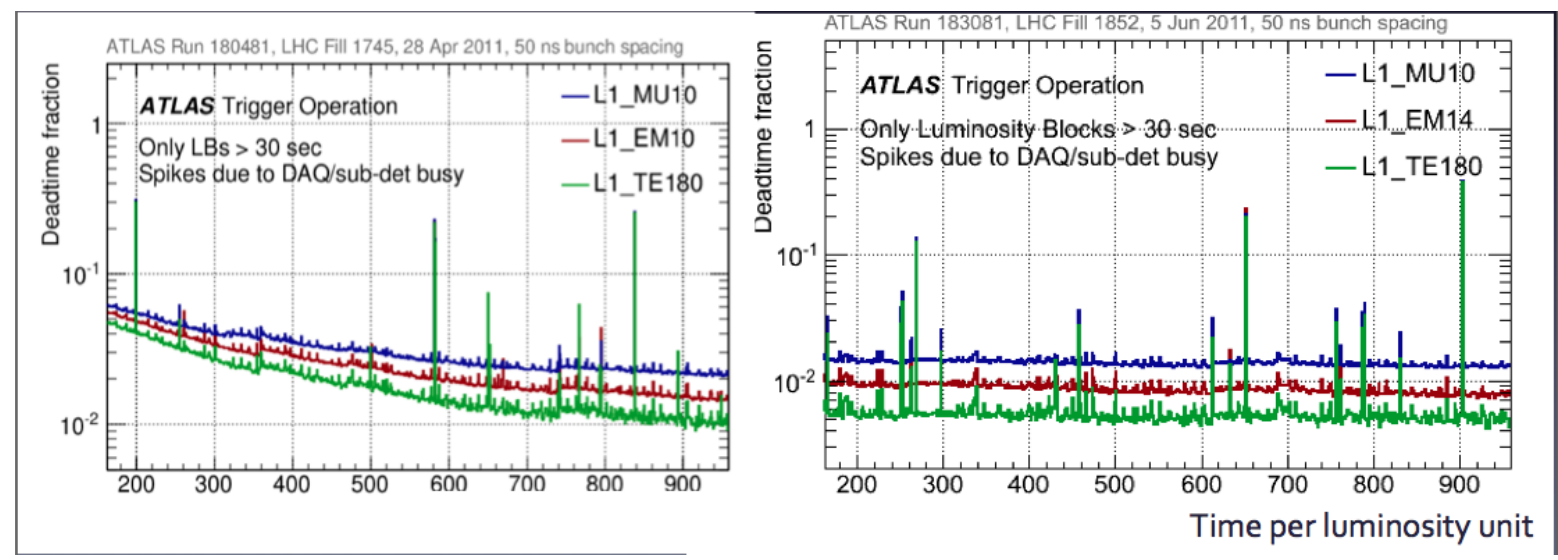

Figure 1: Dead time before (left) and after (right) adjustment of calorimeter noise thresholds[2].

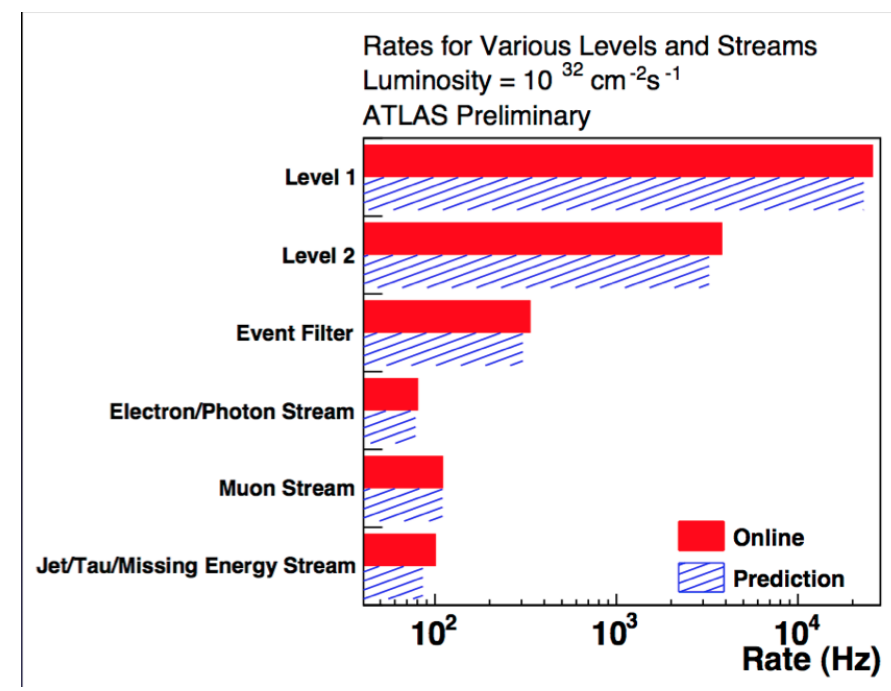

Figure 2: Comparison of trigger rate predictions with observations made online for selected triggers[2].

\section{Conclusion}

The ATLAS TDAQ system has been successfully deployed and commissioned, operating over seven orders of magnitude rise in luminosity since the start of data taking in 2010, whilst maintaining stable data-taking efficiency. Higher thresholds and tighter selections are developing to cope with the increasing LHC demands.

\section{References}

[1] The ATLAS Collaboration, 'Performance of the ATLAS Trigger System in 2010', CERN-PH-EP-2011-078.

[2] https://twiki.cern.ch/twiki/bin/view/AtlasPublic/TriggerOperationPublicResults 